\title{
Dynamic Memory Interval Test vs. Interprocedural Pointer Analysis in Multimedia Applications
}

\author{
ESTHER SALAMÍ and MATEO VALERO \\ Universitat Politècnica de Catalunya (UPC)
}

\begin{abstract}
Techniques to detect aliasing between access patterns of array elements are quite effective for many numeric applications. However, although multimedia codes usually follow very regular memory access patterns, current commercial compilers remain unsuccessful in disambiguating them due mainly to complex pointer references. The Dynamic Memory Interval Test is a runtime memory disambiguation technique that takes advantage of the specific behavior of multimedia memory access patterns. It evaluates whether or not the full loop is disambiguated by analyzing the region domain of each load or store before each invocation of the loop.

This paper provides a detailed evaluation of the approach, compares it against an advanced interprocedural pointer analysis framework, and analyzes the possibility of using both techniques at the same time. Both techniques achieve similar speedups separately (1.25X in average for a 8 -issue width architecture). Furthermore, they can be used together to improve performance (reaching an average speed-up of 1.32X). Results also confirm that memory disambiguation is a key optimization to exploit the available parallelism in multimedia codes, especially for wide-issue architectures (1.50X average speed-up when scaling from 4 - to 12 -issue width in contrast to a low $1.10 \mathrm{X}$ for the baseline compiler).
\end{abstract}

Categories and Subject Descriptors: D.3.4 [Programming Languages]: Processors-Compilers, Optimization

General Terms: Languages, Performance

Additional Key Words and Phrases: Memory disambiguation, multimedia, VLIW

\section{INTRODUCTION}

To make effective use of VLIW processors, the compiler must be able to exploit instruction-level parallelism (ILP). However, ambiguous memory dependences often limit the ability of the compiler to perform code optimizations. If there is any possibility that two memory operations ever reference the same memory location, the compiler must place dependence arcs between them to

Author's address: E. Salamí and M. Valero. Departament d'Arquitectura de Computadors, Universitat Politècnica de Catalunya, Barcelona, Spain; email: \{esalami,mateo\}@ac.upc.es.

Permission to make digital or hard copies of part or all of this work for personal or classroom use is granted without fee provided that copies are not made or distributed for profit or direct commercial advantage and that copies show this notice on the first page or initial screen of a display along with the full citation. Copyrights for components of this work owned by others than ACM must be honored. Abstracting with credit is permitted. To copy otherwise, to republish, to post on servers, to redistribute to lists, or to use any component of this work in other works requires prior specific permission and/or a fee. Permissions may be requested from Publications Dept., ACM, Inc., 1515 Broadway, New York, NY 10036 USA, fax: +1 (212) 869-0481, or permissions@acm.org.

(C) 2005 ACM 1544-3566/05/0600-0199 $\$ 5.00$ 
ensure they are executed in sequential order. Both static and dynamic memory disambiguation approaches have been proposed in the literature to determine if dependence actually exists for a pair of ambiguous memory references.

Static dependence analysis attempts to solve the ambiguity at compile-time. Much work has been done to deal with multidimensional arrays and complex array subscripts [Goff et al. 1991; Maydan et al. 1991; Feautrier 1991; Paek et al. 1998]. However, these techniques are ineffective when the access pattern is nonlinear or when some essential information, such as loop bounds, is not known at compile-time.

Pointer dereferencing is also one of the most important impediments to dependence analysis. Although proposed interprocedural-analysis techniques provide good pointer disambiguation, especially for pointer-intensive applications, such as those of SPECint, they often increase compilation time and memory requirements.

On the other hand, dynamic-memory disambiguation determines at runtime whether two memory operations reference the same location. The compiler provides different execution paths, and at runtime it is determined which one must be followed, depending on the existence or not of the dependence.

Multimedia applications share different traits with both numerical and integer applications. As in numerical applications, media programs make extensive use of multidimensional data structures with relatively simple patterns. As in integer applications, media applications make extensive use of pointers (since $\mathrm{C}$ and $\mathrm{C}++$ are the languages of choice of media developers), sometimes with several levels of indirection to match the media structures of standardized protocols. At the same time, media applications differ from these two wide fields in the characteristics of the data processing. Media applications are streaming, that is, even if there are hard-to-deal-with control and data dependences in the computation, typical media kernels process one or more input streams of data to produce one or more output streams. Moreover, the input and output streams are typically disjointed regions.

The Dynamic Memory Interval Test (DMIT) [Salamí et al. 2002] is a runtime memory-disambiguation technique that makes sense in the context of media applications, or other kind of programs where input and output data streams are usually disjointed. Disambiguation can be easily determined by dynamically analyzing the region domain of every load and store on each invocation of a loop. Significant gains are obtained at nearly no cost and without the inherent complexity of pointer analysis techniques.

This paper focuses on performing a thorough study of the Dynamic Memory Interval Test. As well as providing a more detailed evaluation of the technique, there are two major contributions over previous work. First, we demonstrate that our technique achieves speedups comparable to those of an interprocedural pointer-analysis algorithm. Second, we show that both techniques are not exclusive and can be used together to improve performance.

The rest of the paper is organized as follows. Section 2 gives an overview of pointer-analysis and runtime-dependence tests and justifies a dynamicmemory disambiguation method based on detecting disjointed streamed accesses. Section 3 describes the DMIT technique, including the implementation 
of the algorithm and a code example. Section 4 describes the selected benchmarks and the compilation and simulation framework used for the study. Next, Section 5 presents empirical results, both at the loop-level detail and at the application level, and compares them with those obtained with interprocedural pointer analysis. It also evaluates the option of using a combination of both techniques to improve performance. Finally, Section 5 summarizes the main conclusions.

\section{BACKGROUND}

\subsection{Pointer Analysis}

Pointer-alias analysis attempts to determine at compile-time when two pointer expressions refer to the same memory location. Due to the undecidability of this static analysis [Landi 1992; Ramalingam 1994], existing approaches offer a trade-off between efficiency and precision.

A pointer-analyses algorithm can be classified as flow-sensitive if they use control-flow information during the analysis. On the other hand, it is contextsensitive if it distinguishes different caller contexts for a common callee. Several approaches are flow-sensitive and context-sensitive [Landi and Ryder 1992; Choi et al. 1993; Emami et al. 1994; Wilson and Lam 1995]. By contrast, other algorithms are flow-insensitive [Andersen 1994; Steensgaard 1996; Shapiro and Horwitz 1997]. One approach proposes a tunable pointer-analysis framework for handling structures in the presence of casting [Yong et al. 1999].

Qualitative comparisons among algorithms are difficult due to varying infrastructure, benchmarks, and performance metric. An empirical comparison of the effectiveness of different pointer algorithms on $\mathrm{C}$ programs can be found in Hind and Pioli [2000].

The pointer analysis used in this paper employs a flow-insensitive, but context-sensitive, interprocedural algorithm that can handle all $\mathrm{C}$ features. Pcode-interprocedural analysis [Gallagher 1995] determines what dependences exist with regard to global variables across function boundaries. This analysis also performs intraprocedural-pointer disambiguation and dependence analysis, gathers alias and side effect information, and identifies targets of indirect function calls. This information is then merged back into the Pcode and is used by subsequent stages of the compilation.

\subsection{Runtime Dependence Tests}

Dynamic data-dependence tests can be used at runtime to check whether two references access the same location. Dynamic-memory disambiguation techniques usually require significantly less compile-time investment than static approaches, especially in languages such as $\mathrm{C}$, that require interprocedural analysis to provide high accuracy. Dynamic approaches are also more accurate than static ones, as they know the exact memory address being accessed by each reference during program execution.

The obvious downside of runtime tests is the overhead they introduce into the program. They usually require the insertion of extra instructions to check 
dependences. Some approaches also require new instructions and/or additional hardware support.

Whether static, dynamic, or a combination of both is better depends on the particular kind of application being targeted and on the desired trade-off between performance and cost. Gallagher et al. [1994] investigate the application of both static- and dynamic-memory disambiguation approaches and provide a quantitative analysis of the trade-offs between the two approaches.

Nicolau [1989] first introduced runtime-memory disambiguation. He proposed a software data-speculation technique that inserts explicit address comparisons and conditional branch instructions that allow memory-flow dependences to be removed safely. Huang et al. [1994] proposed speculative disambiguation, a combined hardware and software technique to allow aggressive code reordering using predicated instructions.

A different point of view is to consider the problem of deciding if a loop is fully disambiguated or not, that is, determining whether or not there is a dependence in any iteration [Bernstein et al. 1994]. The Privatizing Do All Test [Rauchwerger and Padua 1994], for instance, identifies fully parallel loops at runtime and dynamically privatizes scalars and arrays; significant speed-ups were obtained on Fortran loops running on multiprocessor architectures.

Other sophisticated approaches exist that produce predicates that may be used either at compile-time or at runtime, depending on whether there is enough information available [Moon and Hall 1999; Pugh and Wonnacott 1998].

The runtime test proposed in this paper identifies a type of ambiguous dependences commonly found in multimedia applications that can be easily eliminated with a simple test at runtime. It requires no instructions or hardware support, and thus can be applied to any existing architecture.

\subsection{Memory Disambiguation for Multimedia Loops}

Array references in multimedia applications usually follow strided and very simple access patterns. Figure 1 summarizes the kind of loops commonly found in these codes. The loop in Figure 1(a) operates over one or several streams to produce another one, and no memory dependence exists. Nevertheless, when these arrays are accessed through pointers, as is usual in media codes, an accurate interprocedural pointer analysis is required to ensure that no aliasing occurs. Such techniques are not generally included in common commercial compilers, so they must be conservative and place dependence arcs between the memory operations to ensure correctness.

Output dependences (dependences between two stores) and antidependences (when a load precedes a dependent store) usually have little impact on the generated code, but flow dependences (when a store precedes a dependent load) tend to be a severe restriction for the compiler. In the example, the potential loop-carried dependences from the store of $A$ in the iteration $i$ to the loads of $B$ and $C$ in the iteration $i+1$ restrict aggressive techniques, such as modulo scheduling [Rau 1995].

In loop Figure 1(b), the input and output streams coincide. However, loopcarried dependences do not exist in this case either, as loads from iteration 


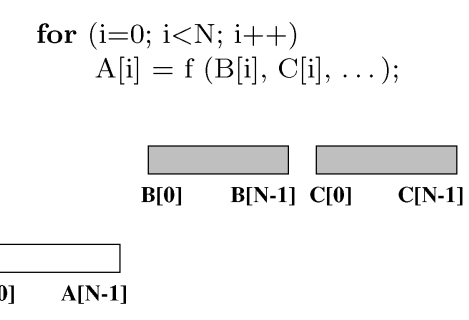

(a) Disjointed

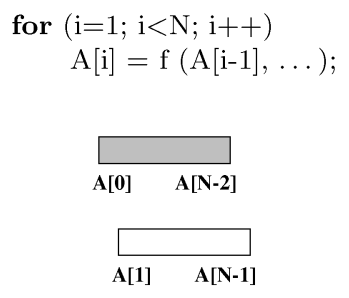

(c) Recurrence

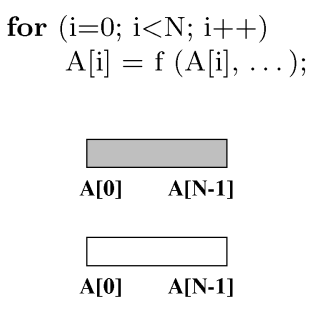

(b) Coincident

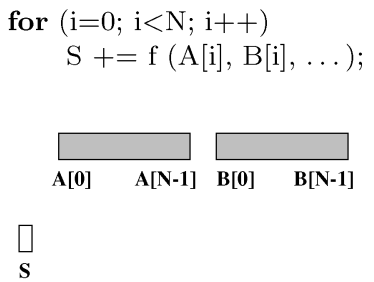

(d) Reduction

Fig. 1. Typical multimedia memory access patterns.

$i+1$ never to refer to the same memory location as stores from iteration $i$. The opposite case is shown in Figure 1(c), where there is a recurrence with the distance one. In this case, loads from iteration $i+1$ must not precede the stores from iteration $i$. The last case shown in Figure 1(d) shows a loop that operates on array elements and accumulates the result on a scalar variable $S$. A register will probably be assigned to the scalar and dependences between two loads (input dependences) are not a problem, so there are not ambiguous memory dependences in that case.

In numerical applications, the identification of the array elements accessed by a particular reference is important for compiler optimizations. In contrast, we observe that memory references on media loops are always dependent or nondependent at all. In other words, we have found that in multimedia we have two main kinds of stream behavior: one where all the input and output streams are totally independent, and other where the streams have recurrences between themselves. A cost-effective approach to perform memory disambiguation would just need to determine, for every loop, which case we are facing.

The concept of calculating nonintersecting data access ranges was probably first explored in Blume and Eigenmann [1994], and later expanded by Paek et al. [1998], to handle symbolic array subscripts in scientific applications at compile-time. Our work differs from previous work by observing that, in multimedia loops, the indexing functions are so simple that data access ranges can be easily computed at runtime. The intersecting or nonintersecting of these ranges cannot be determined at compile-time mainly due to the use of pointers, but not because of the complexity of the indexing functions.

References with similar array index functions that differ only in the constant term \{like $A[i], A[i+1]$ and $A[i+2]$ in the code example in Figure 2(a)\} 


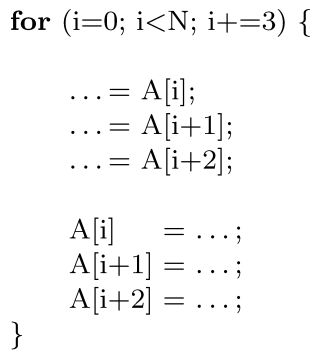

(a) C code

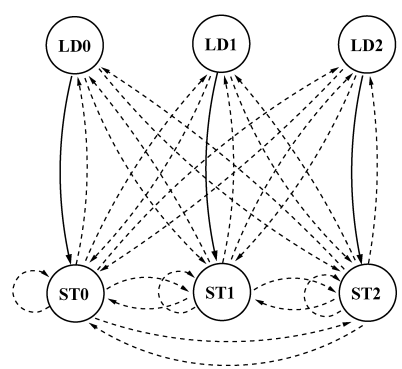

(b) Dependence graph

Fig. 2. Example of reference groups.

are also quite frequent in multimedia loops. These memory-access patterns are known as uniformly generated references [Wolf and Lam 1991] or reference groups [Carr et al. 1994]. In a reference group, two references with different constant terms are independent inside each iteration. Moreover, if the stride of the variable term is greater or equal to the maximum difference between the constant terms, loop-carried dependences do not exist either. Thus, all dashed arcs in the dependence graph in Figure 2(b) can be safely eliminated. We have added to our compiler the ability to detect and disambiguate reference groups.

Indirect references, nonlinear array indices, or linked lists of data are not common in multimedia loops. The main limitation to our approach is the use of nonstreaming (sparse) data structures to perform computations via memory tables. Most of the algorithms used in current standards have a streaming nature. Nevertheless, due to the intrinsic significance of most multimedia algorithms, there has been a great effort focused on reducing the overall number of required operations. Unfortunately, this effort has been oriented toward low-end DSP architectures, thus focusing on reducing the number of operations or, more specifically, the number of high latency operations such as multiplications, rather than on making explicit the potential ILP of the algorithms [Salamí et al. 1999].

\section{DYNAMIC MEMORY INTERVAL TEST}

\subsection{Description}

The Dynamic Memory Interval Test (DMIT) is a software-only mechanism based on the multimedia memory-access patterns described in Section 2.3. The compiler generates both disambiguated and nondisambiguated versions of the loop, and inserts a simple test block that decides at runtime which one must be executed (see Figure 3). This decision is made by computing and comparing the lower and upper memory addresses that will be accessed by each stream. Complex pointer references or unknown parameters, such as loop bounds, prevent the compiler from making the decision at compile-time.

The test block is executed once on each invocation of the loop. In most cases, reducing the length of the disambiguated loop schedule will compensate for the already low overhead involved in the calculation of the intervals. Otherwise, 

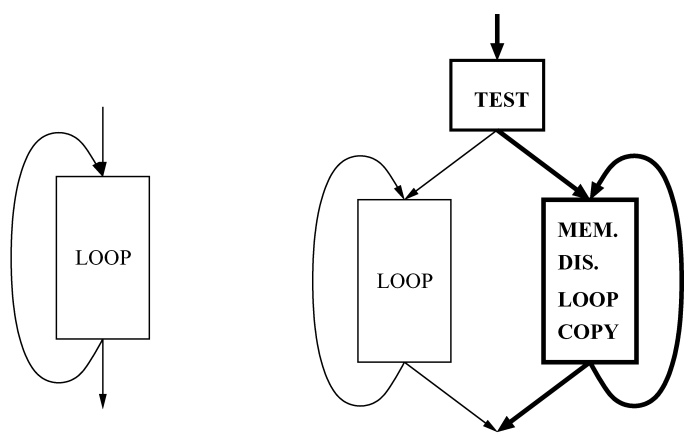

(a) Original control flow

(b) New control flow

Fig. 3. Loop duplication.

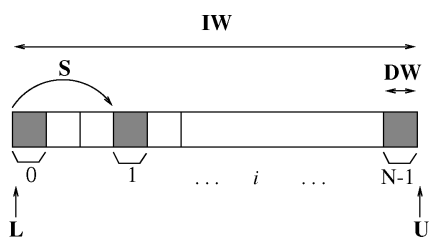

(a) Single memory reference

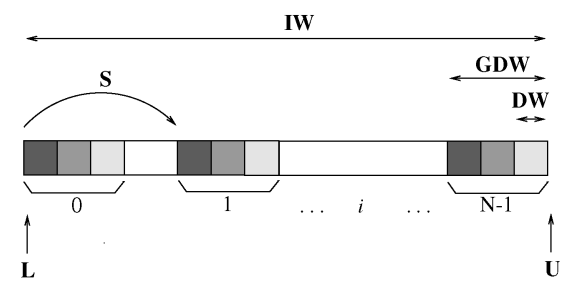

(b) Memory reference group

$$
\begin{array}{lll}
\text { L: lower bound } & \text { DW: data width } & \text { N: number of iterations } \\
\text { U: upper bound } & \text { GDW: group data width } & \text { S: stride } \\
& \text { IW: interval width } &
\end{array}
$$

Fig. 4. Dynamic memory interval.

the penalty introduced by this block (if it turns out that the original loop is executed) is minimal and has no relevant impact on performance.

\subsection{Dynamic Memory Interval}

We define the Dynamic Memory Interval (DMI) of a memory reference as the memory space delimited by the lower and upper locations accessed by that operation during one invocation of the loop. Figure 4(a) shows the DMI of a stride $S$ memory reference inside a loop of $N$ iterations. The shadow boxes represent the memory addresses that are actually accessed. Following this terminology, if we are able to prove before entering a loop that the DMIs of two references do not overlap, we can ensure that they are independent in that invocation. Note that scalar references are also included in this definition, as they are, in fact, stride-zero references. 
In the case of a reference group, if we were to apply the test between each pair of references inside the group, they would fail, as their individual DMIs overlap. When the compiler detects a reference group, it builds only one DMI for the entire group. The stride of the reference group is the same as that of each individual reference. The group data size is the size of the memory space traveled by the different references of the group on each iteration (see Figure 4(b)).

The following notation is used in the figures and algorithm description:

$-T B$ : test block

$-L B$ : loopbody

-Ref: memory reference

$-A R_{i}$ : address register of $R e f_{i}$

$-S_{i}: \quad$ stride of $\operatorname{Ref}_{i}$ (in bytes)

$-D W_{i}$ : data size of $\operatorname{Ref}_{i}$ (in bytes)

$-N$ : number of loop iterations in current invocation

$-L_{i}$ : the lowest location referred by $R e f_{i}$ in current invocation

$-U_{i}$ : the next location to the highest one referred in current invocation

$-I W_{i}:$ size of the memory region between $L_{i}$ and $U_{i}$

$-E_{i j}:$ name for dependence arc from $\operatorname{Ref}_{i}$ to $R e f_{j}$

\subsection{Algorithm Implementation}

3.3.1 Main Algorithm. This section describes the main features of the implementation. The main algorithm is shown in Figure 5. Solving ambiguous memory dependences becomes especially profitable to software-pipelining techniques, such as modulo scheduling, where just one ambiguous loop-carried memory dependence is enough to prevent the compiler from overlapping different iterations of the loop. In this study, we consider only loops that are targeted with modulo scheduling by the baseline compiler.

Memory dependences in which one of the two references is neither strided nor loop invariant are discarded, as their DMIs cannot be computed before each execution of the loop. Dependences between references, which can be statically determined to refer the same location, are also excluded, as they are definitely dependent. If any pairs of references still remain in the list, the compiler duplicates the loop and inserts the test block. This block contains the operations needed to test each of the selected dependences. Finally, dependences in the list are removed in the disambiguated loop version.

The length of the test block should be controlled not only because of performance, but also because it increases the register pressure. For our study, we have used a simple heuristic that limits the maximum number of dependences to be tested. Loop duplication is also avoided if it does not reduce the minimum initiation interval (MII) for modulo scheduling.

3.3.2 The Test Block. Suppose an ambiguous memory dependence exists between two references whose DMIs are $\left[L_{i}, U_{i}\right)$ and $\left[L_{j}, U_{j}\right)$. Then, to ensure 


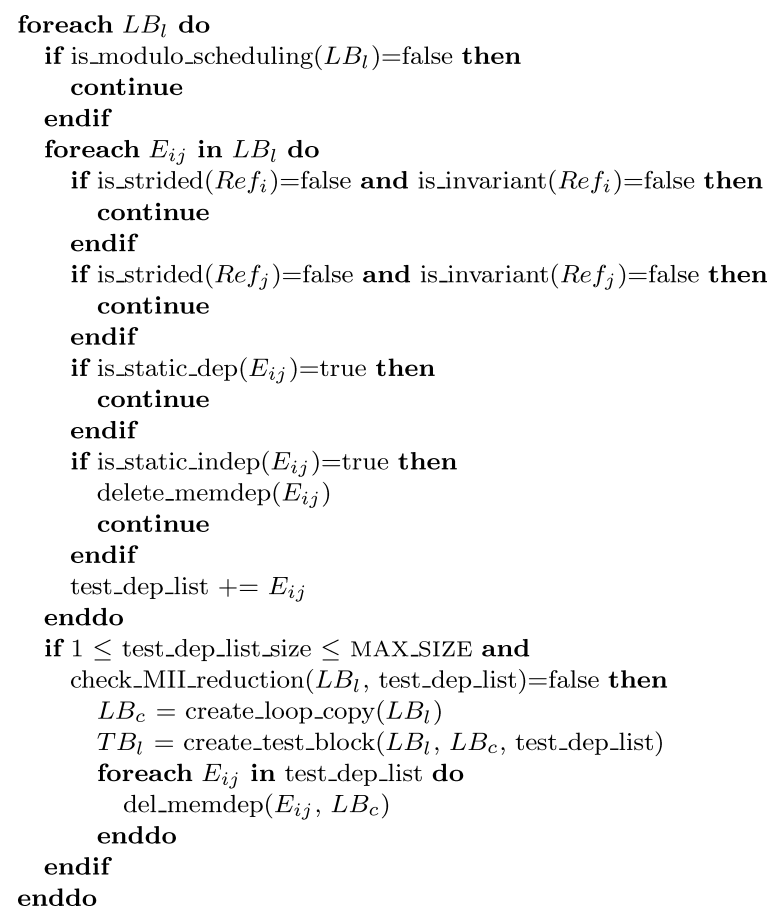

Fig. 5. Main algorithm.

they are disjointed intervals, we must test that:

$$
L_{j} \geq U_{i} \quad \text { or } \quad L_{i} \geq U_{j}
$$

where $L_{k}$ and $U_{k}$ can be computed in this way:

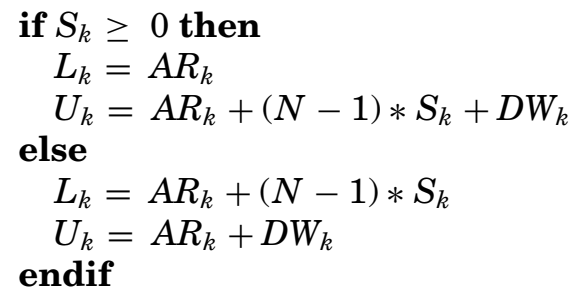

Note that it handles both positive and negative strides. In case of a reference group, the group data width is used instead of the data width. The group data width can be computed as the difference between the highest and lowest constant terms of the array index functions plus the data width. The stride and the data width are usually known at compile-time, while the address register and, sometimes, the number of iterations are not.

The steps to create the test block are summarized in Figure 6. The insert previous_ops function takes into account the case in which the address register is defined inside the loopbody before being used by the memory operation. This is the case when the value of the register $A R$ before entering the loop is not the value it will have when the memory operation is executed in the first iteration. 


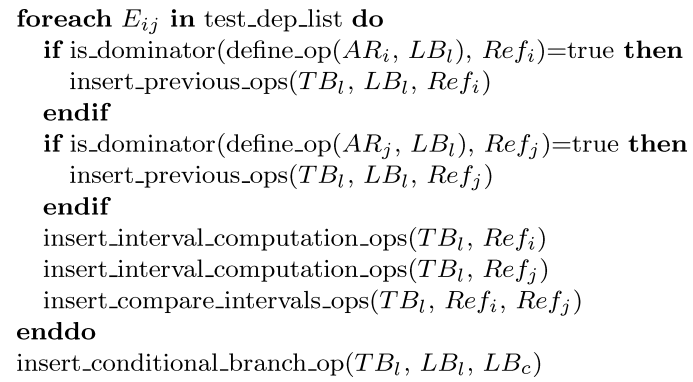

Fig. 6. Test block generation algorithm.

In that case, the compiler must also insert an equivalent copy of the define operation before the bounds computation in order to get the right value of $A R$. In this copy, the register is renamed to avoid modifying the real value. Note that insert_previous_ops is a recursive function, as it must now ensure data dependences are maintained for each operand of the define operation. The function insert_interval_computation_ops creates the low level products and additions to compute $L$ and $U$, and insert_compare_intervals_ops inserts the operations to compare these limits. Finally, insert_branch_op inserts the conditional branch.

At first sight, for each pair of intervals to be compared, we would need two products, six adds and two compare operations. Such a quantity of operations could become prohibitive as the number of dependences to be tested increases. However, they are actually reduced if we take into account some trivial considerations. For instance, intervals with the same stride share a single product. Also, if a memory reference must be compared with more than one other, the interval bounds are computed just once, so that only the compare operations are added.

\subsection{Code Example}

As an example, we will show the test block created by the compiler for the innermost loop of the color conversion function in the JPEG encoder application. For each pixel of a row, the three color components $(R, G$, and $B)$ are loaded each iteration [see $\mathrm{C}$ code in Figure 7(a)]. These color components are one byte sized and are interleaved stored in memory:

$$
R_{0} G_{0} B_{0} R_{1} G_{1} B_{1} R_{2} G_{2} B_{2} R_{3} \ldots
$$

This code shows the relevance of detecting reference groups. Without grouping, the three inp loads would produce three DMIs with stride three and data width one byte, and each one should be compared with the DMIs of other references. However, if the compiler detects the group, it considers just one interval with stride three and group data size three [see Figure 7(b)], saving an important number of arithmetic and compare operations. The effect is even greater for loops with large reference groups, such as the DCT computation, where the size of the groups is eight.

The code of the test block inserted by the compiler to disambiguate the three loads and the three stores of the color conversion loopbody is given in 


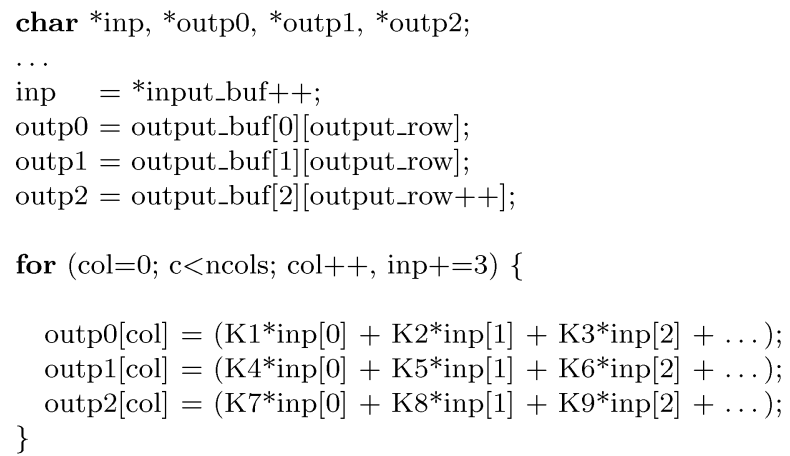

(a) C code fragment

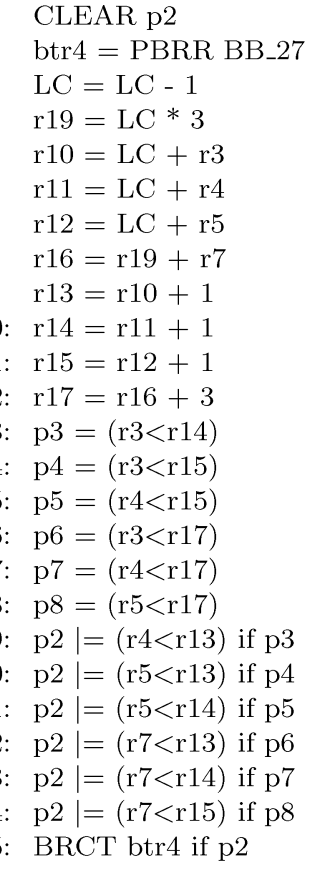

(c) Test block code

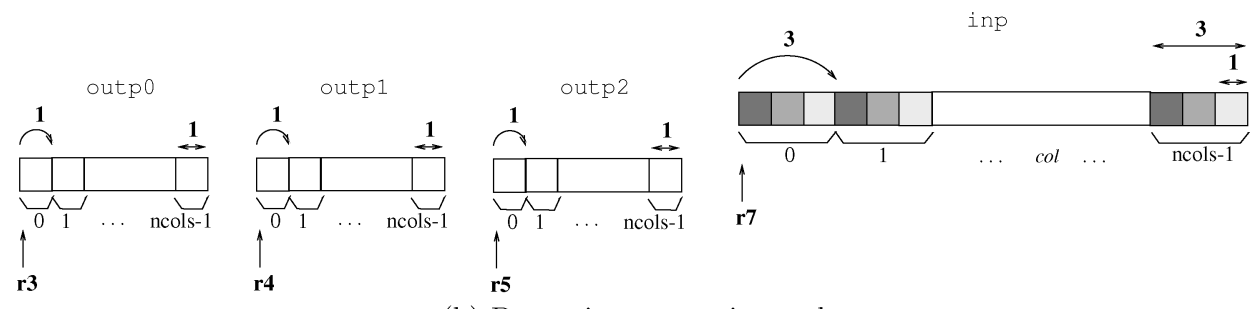

(b) Dynamic memory intervals

Fig. 7. Example of a test block code generated by the compiler.

Figure 7(c). Let us assume that registers $r 3, r 4, r 5$, and $r 7$ are the address registers pertaining to outp 0 , outp 1 , outp 2 , and inp, respectively, and the control register $L C$ (loop counter) contains the number of iterations. Operations from 3 to 12 are then inserted to compute the interval bounds $(r 3, r 4, r 5$, and $r 7$ are the lower bounds and $r 13, r 14, r 15$, and $r 17$ the upper ones).

Once the interval bounds are computed, the compiler introduces the operations needed to check whether they overlap (operations 1 and from 13 to 24). To support predicated execution, the HPL-PD architecture [Kathail et al. 2000] provides 1-bit predicate register files and a rich set of compare-to-predicate operations which set predicate registers. We make use of these capabilities to generate the code of the test block. In the example, predicate registers are denoted as $\mathrm{p} n$. The OR-compare operations [e.x., $p 2 \mid=(r 7<r 15)$ if $p 8$ ] write 
a 1 into the destination register ( $p 2$ ) only if both the predicate input ( $p 8$ ) and the result of the comparison are true. Otherwise, they leave the destination unchanged.

The conditional branch is performed in two steps. First, the prepare-tobranch (PBRR) operation loads the target address into a branch-target register (btrn). Second, the branch-conditional (BRCT) operation branches to the address contained in the btrn operand if the branch condition (available in the specified predicate) is true. In the example, the branch-target register btr 4 holds the address of the nondisambiguated loop version and operation 25 branches to it if the result of the comparisons is true.

\section{METHODOLOGY}

\subsection{Benchmarks}

For our study we focus on eight representative programs of image, audio, and video, all from the UCLA Mediabench suite [Lee et al. 1997]:

- jpeg_enc and jpeg_dec: JPEG compression and decompression standard for either gray-scale and color digital images.

-mpeg2_enc and mpeg2_dec: encoder and decoder of the MPEG2 standard for high-quality digital video transmission.

- gsm_enc and gsm_dec: encoder and decoder of the European GSM 6.10 standard for full-rate speech transcoding.

—epic_enc and epic_dec: image compression and decompression.

\subsection{Compilation and Simulation Framework}

We have implemented the DMIT technique in the Trimaran compiler and evaluated it for a VLIW architecture with guarded execution. Trimaran [Lab. et al. 1998] is a compiler infrastructure that supports state-of-the-art research in compiling for ILP architectures. It is comprised of Impact [Hwu et al. 1995], a compiler front-end for $\mathrm{C}$, which performs machine-independent optimizations, Elcor [Aditya et al. 1998], which performs machine-dependent optimizations, instruction scheduling, and register allocation, and a cycle-level simulator of the parameterized architecture. To expose sufficient ILP, it makes use of advanced techniques such as Superblock [Hwu et al. 1993] or Hyperblock [Mahlke et al. 1992] formation.

Our baseline is the original compiler memory-disambiguation model, which is quite representative of current commercial compilers. It only performs intraprocedural analysis on low-level code. We have incorporated a new compilation module into the Elcor back-end to do loop disambiguation. Loop disambiguation is performed at the intermediate code level just before any scheduling or register allocation.

To perform Interprocedural Pointer Analysis (IPA), we use the Pcodeinterprocedural analysis [Gallagher 1995]. Pcode is the first module of the Impact front-end, so both techniques are evaluated using the same compilation and simulation framework. 
Table I. Coverage

\begin{tabular}{|c|c|c|c|c|c|c|}
\hline \multirow[b]{2}{*}{ Benchmark } & \multicolumn{2}{|c|}{ Innermost } & \multicolumn{2}{|c|}{ +Modulo scheduling } & \multicolumn{2}{|c|}{+ With stores } \\
\hline & \#loops & $\%$ cycles & \#loops & $\%$ cycles & \#loops & $\%$ cycles \\
\hline jpeg_enc & 6 & 51.63 & 6 & 51.63 & 6 & 51.63 \\
\hline jpeg_dec & 4 & 69.43 & 4 & 69.43 & 4 & 69.43 \\
\hline mpeg2_enc & 13 & 68.82 & 13 & 68.82 & 5 & 6.15 \\
\hline mpeg2_dec & 11 & 35.86 & 9 & 32.38 & 8 & 31.36 \\
\hline gsm_enc & 11 & 71.99 & 10 & 71.14 & 7 & 48.23 \\
\hline gsm_dec & 7 & 89.91 & 3 & 8.15 & 3 & 8.15 \\
\hline epic_enc & 13 & 67.50 & 8 & 52.50 & 2 & 15.24 \\
\hline epic_dec & 12 & 76.68 & 7 & 60.78 & 5 & 58.49 \\
\hline sum/average & 77 & 66.48 & 60 & 51.85 & 40 & 36.09 \\
\hline
\end{tabular}

We have evaluated 4-, 8- and 12-issue width VLIW architectures. In order to support the high computational demand of multimedia applications, our configurations are quite aggressive in the number of arithmetic functional units. The number of integer functional units is equal to the width of the architecture.

The first-level data cache is a $64 \mathrm{~KB}$, 4-way set associative pseudomultiported cache; the second level is a $1024 \mathrm{~K}, 2$-way set associative cache. Latencies are 3 cycles for the first-level cache, 12 cycles for the second level, and 500 cycles to main memory. We have not simulated the instruction cache, since our benchmarks have small-instruction working sets. The compiler schedules all memory operations, assuming they hit in the first-level cache and the processor is stalled at runtime in case of a cache miss or a bank conflict.

\section{EXPERIMENTAL RESULTS}

\subsection{Dynamic Memory Interval Test Evaluation}

5.1.1 Coverage. Current implementation of the DMIT only applies to innermost modulo scheduling loops. This means that it cannot disambiguate multidimensional array accesses (except when the innermost loop has been fully unrolled). The algorithm could be extended to work on nested loops. However, this would increase the implementation complexity, which is one of the main advantages of our approach.

Moreover, there are some loops that have no potential to be disambiguated, as they contain no store operations. This is the case, for example, of the main loop in the motion estimation algorithm of the mpeg2 enc, where the sum of absolute differences is computed for two arrays of $16 \times 16$ elements.

Table I shows the number and fraction of cycles of innermost, modulo scheduling, and modulo-scheduling loops with store operations for each application when they are executed in the 4-issue width architecture. Only loops that account for more than $0.5 \%$ of the overall program cycles are included. Loops in the last column are the input candidates to DMIT.

Only two of the eight benchmarks show low coverage. The rest of the benchmarks present a sufficient number of loops to improve performance as a result of including memory disambiguation. Especially interesting are jpeg_enc, jpeg_dec, and epic_dec, which show a high potential for improvement 
Table II. DMIT. Loop-Level Analysis for the 8-Issue Width Architecture ${ }^{a}$

\begin{tabular}{|c|c|c|c|c|c|c|c|}
\hline Benchmark & Loop name & $\%$ сус & $\begin{array}{c}\text { Old LB } \\
\text { OPC }\end{array}$ & $\begin{array}{c}\text { New LB } \\
\text { OPC }\end{array}$ & $\begin{array}{c}\text { Test } \\
\text { cyc/inv }\end{array}$ & $\begin{array}{c}\text { Test } \\
\% \text { pass }\end{array}$ & $\begin{array}{l}\text { Loop } \\
\text { SP }\end{array}$ \\
\hline \multirow[t]{6}{*}{ jpeg_enc } & _rgb_ycc_c.5 & 7.61 & 2.50 & 7.00 & 12 & 100 & 2.79 \\
\hline & forward_D.9 & 31.75 & 1.03 & 7.80 & 9 & 100 & 6.69 \\
\hline & forward_D.6 & 4.13 & 1.29 & 4.50 & 9 & 100 & 2.03 \\
\hline & _h2v2_down.4 & 2.72 & 1.90 & 6.33 & 8 & 100 & 3.30 \\
\hline & .jpegfdct. 3 & 2.31 & 4.80 & 7.38 & - & - & 1.54 \\
\hline & _.jpeg_fdct.5 & 2.31 & 4.90 & 7.54 & 5 & 100 & 1.48 \\
\hline \multirow[t]{4}{*}{ jpeg_dec } & _ycc_rgb_c.5 & 31.02 & 2.53 & 8.00 & 13 & 100 & 3.16 \\
\hline & _h2v2_fanc. 8 & 17.89 & 2.27 & 6.25 & 9 & 100 & 2.73 \\
\hline & jpeg_idct.3 & 9.37 & 4.74 & 7.53 & 9 & 100 & 1.50 \\
\hline & _jpeg_idct.5 & 9.37 & 5.44 & 5.44 & - & - & 1.00 \\
\hline \multirow[t]{5}{*}{ mpeg2_enc } & _quant_int.6 & 1.34 & 0.58 & 7.25 & 8 & 0 & 1.00 \\
\hline & _quant_non.3 & 3.08 & 0.63 & 7.75 & 8 & 0 & 1.00 \\
\hline & _iquant_in.5 & 0.64 & 1.04 & 6.25 & 8 & 0 & 0.99 \\
\hline & iquant_no.5 & 1.76 & 1.11 & 7.75 & 8 & 0 & 1.00 \\
\hline & _add_pred_.4 & 0.64 & 1.40 & 3.50 & 7 & 100 & 1.91 \\
\hline \multirow[t]{8}{*}{ mpeg2_dec } & Add_Block.31 & 12.68 & 1.53 & 1.53 & - & - & 1.00 \\
\hline & Add_Block.42 & 5.03 & 1.31 & 1.31 & - & - & 1.00 \\
\hline & _Fast_IDCT.3 & 3.77 & 3.69 & 7.38 & - & - & 2.00 \\
\hline & Fast_IDCT.5 & 4.78 & 4.64 & 7.65 & 5 & 100 & 1.61 \\
\hline & form_comp.18 & 1.32 & 1.25 & 5.00 & 7 & 100 & 2.22 \\
\hline & fform_comp.58 & 2.25 & 1.43 & 5.00 & 7 & 100 & 2.42 \\
\hline & form_comp.38 & 0.63 & 1.43 & 5.00 & 7 & 100 & 2.53 \\
\hline & fform_comp.73 & 0.57 & 2.00 & 5.33 & 7 & 100 & 2.16 \\
\hline \multirow[t]{7}{*}{ gsm_enc } & _Gsm_Coder.5 & 1.91 & 1.64 & 6.00 & 8 & 0 & 0.98 \\
\hline & _Calculati.25 & 1.04 & 1.33 & 8.00 & 8 & 100 & 4.56 \\
\hline & _Long_term. 8 & 4.00 & 0.96 & 7.33 & 8 & 100 & 6.46 \\
\hline & Autocorre.42 & 16.40 & 0.82 & 6.92 & 7 & 100 & 8.33 \\
\hline & _Reflectio.52 & 1.52 & 1.22 & 7.14 & 8 & 100 & 3.60 \\
\hline & _Weighting.3 & 4.53 & 2.19 & 7.12 & 9 & 100 & 3.02 \\
\hline & _Short_ter.5 & 22.92 & 3.13 & 5.22 & 9 & 100 & 1.25 \\
\hline \multirow[t]{3}{*}{ gsm_dec } & _Gsm_Decod.5 & 1.00 & 1.40 & 7.00 & 8 & 100 & 3.87 \\
\hline & _Gsm_Long_.16 & 4.01 & 1.15 & 7.67 & 8 & 100 & 5.62 \\
\hline & _Gsm_Long_.24 & 2.96 & 1.40 & 7.00 & - & - & 4.84 \\
\hline epic_enc & reflect1.108 & 0.63 & 5.86 & 5.86 & - & - & 8.03 \\
\hline \multirow[t]{5}{*}{ epic_dec } & _collapse_.191 & 0.94 & 1.50 & 6.00 & 13 & 100 & 3.99 \\
\hline & _collapse_.9 & 10.78 & 1.17 & 1.17 & - & - & 1.00 \\
\hline & _write_pgm. 3 & 2.85 & 1.75 & 7.00 & 8 & 100 & 4.00 \\
\hline & _unquantiz.3 & 11.42 & 1.31 & 7.00 & 8 & 100 & 5.33 \\
\hline & _main.18 & 32.82 & 0.41 & 6.33 & 9 & 100 & 15.33 \\
\hline
\end{tabular}

${ }^{a} O P C$, operations per cycle; $S P$, speed-up; cyc, cycles; inv, invocations; -, no test.

based on coverage issues. It is important to note that our technique adds nearzero overhead over those codes that could not benefit from memory disambiguation, thus overcoming the fact that there exist benchmarks without room for improvement.

5.1.2 Loop-Level Analysis. Table II shows the results of applying DMIT to the candidate loops for the 8-issue width architecture. It includes the percentage of the execution time for the complete application they represent, the operations per cycle rate for the disambiguated and nondisambiguated loopbodies, the 
cycle count per invocation of the test block, how many times the loop passes the test at runtime, and, finally, the speed-up of the loop. Note that, although all memory operations are scheduled assuming the first-level cache latency, stall cycles due to the memory hierarchy (such as, cache misses or bank conflicts) are not taken into account in this table. Thus, real speed-ups and operation per cycle rates would be slightly lower.

As can be seen, the nondisambiguated versions of the loops are not making a good use the processor resources and barely execute an average of 1.57 operations per cycle. On the contrary, the disambiguated versions achieve an average rate of 4.24 operations per cycle.

The test results show that a high percentage of the loop candidates disambiguate (only 5 loops of 32 fail the test), which supports the assumption that media loops are characterized by high amounts of data-level parallelism. Nevertheless, test blocks represent a negligible overhead compared with the rest of the code. Even for the mpeg2_enc, a benchmark without coverage and in which four of the five candidate loops fail the test, there is not a relevant impact in performance. Furthermore, we observe that the result of the test is always the same in all invocations of the loops.

On the other hand, 8 loops of the 40 candidates do not require DMIT. In these cases, simple static disambiguation is enough to determine the dependence or independence of the memory references, without the requirement of a very complex array dependence analysis.

5.1.3 Complete Applications Speed-up. Figure 8 shows the performance speed-up obtained in complete applications for different issue widths, both with and without DMIT. All speed-ups are related to the 4-issue width architecture without DMIT.

Results show that memory disambiguation is a key technique to allow an effective exploitation of the available ILP when we increase the width of the machine. The disambiguated version of the code provides up to $1.38 \mathrm{X}$ (1.17X in average) improvement in performance in the 4-issue width architecture. Furthermore, the original version of code fails to scale adequately when we increase the way of the machine (except for mpeg2_enc, which, as said before, has no memory-aliasing problems). In sharp contrast, the disambiguated versions of code show high performance increases when scaling the reference machine, especially for those benchmarks with high coverage, where we observe up to 1.78X performance improvement.

On the other hand, we have also observed a degradation of the memory behavior in the disambiguated versions. As an effect of increasing the parallelism, memory pressure also increases, and the number of bank conflicts rises up significantly. Moreover, as processor cycles decrease, memory cycles become a greater percentage of the total execution time. The worst case is observed in the epic_dec benchmark: in the 8-issue width baseline, the $27.90 \%$ of the execution time is due to memory stalls. This percentage goes up to $41.39 \%$ in the 8-issue width disambiguated version. These memory offset cycles make the speed-up decrease from an ideal $1.76 \mathrm{X}$ (without processor stalls) to the $1.43 \mathrm{X}$ shown in the graph. 


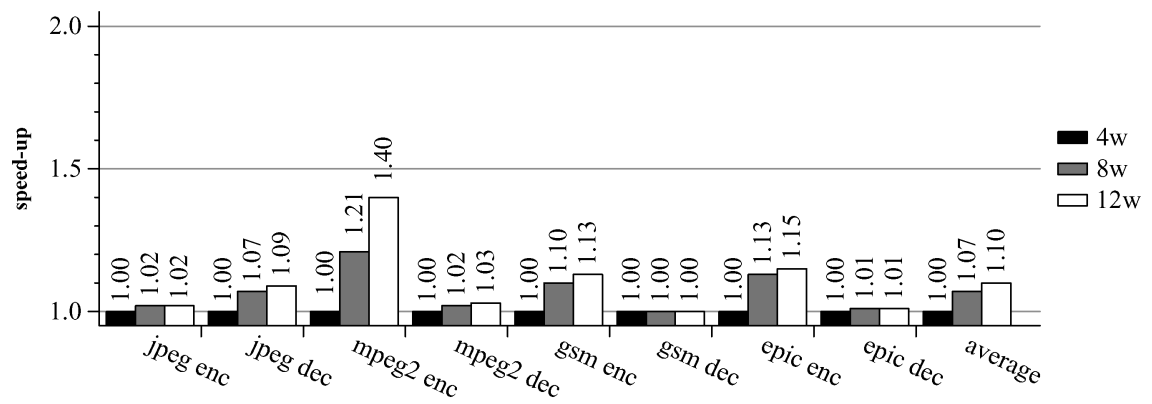

(a) Baseline

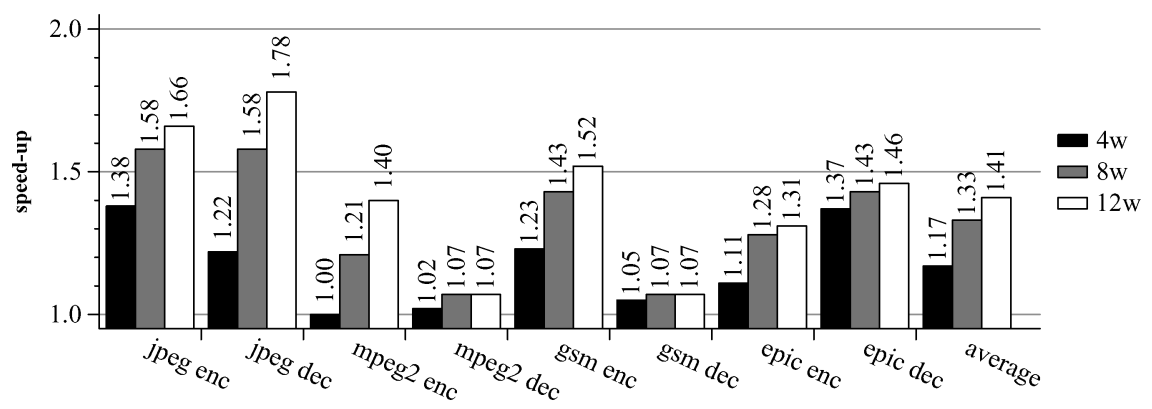

(b) DMIT

Fig. 8. Performance speed-up of 4-, 8- and 12-issue width architectures with and without DMIT over the 4-issue width baseline.

Table III. Code Size Increase

\begin{tabular}{l|c}
\hline Benchmark & \% Code Size Increase \\
\hline jpeg_enc & 0.74 \\
jpeg_dec & 0.72 \\
mpeg2_enc & 0.87 \\
mpeg2_dec & 1.49 \\
gsm_enc & 1.90 \\
gsm_dec & 0.75 \\
epic_enc & 0.30 \\
epic_dec & 1.87 \\
average & 1.08 \\
\hline
\end{tabular}

5.1.4 Code Size Increase. The DMIT involves an increase in code size, but as the optimization is only applied to a minimal part of the static code, the overall impact is negligible. Experimental results show that the duplicated loopbodies have an average size of 34 static operations, and the test block size ranges from 5 to 25 (16 in average) static operations. The percentage increase of the overall code size is shown in Table III. As can be seen, it is less than $2 \%$ for all the benchmarks. 
Table IV. IPA. Loop Level Analysis for the 8-Issue Width Architecture ${ }^{a}$

\begin{tabular}{|c|c|c|c|c|c|c|c|c|c|}
\hline \multirow[b]{2}{*}{ Benchmark } & \multirow[b]{2}{*}{ Base OPC } & \multicolumn{3}{|c|}{ DMIT } & \multicolumn{2}{|c|}{ IPA } & \multicolumn{3}{|c|}{ IPA+DMIT } \\
\hline & & $\# \mathrm{~T} / \mathrm{L}$ & $\mathrm{OPC}$ & SP & $\mathrm{OPC}$ & SP & $\# \mathrm{~T} / \mathrm{L}$ & $\mathrm{OPC}$ & $\mathrm{SP}$ \\
\hline jpeg_enc & 1.66 & $5 / 6$ & 6.31 & 3.79 & 5.66 & 3.40 & $1 / 6$ & 6.66 & $\overline{4.01}$ \\
\hline jpeg_dec & 3.17 & $3 / 4$ & 6.71 & 2.12 & 5.04 & 1.59 & $1 / 4$ & 7.25 & 2.29 \\
\hline mpeg2_enc & 0.84 & $5 / 5$ & 0.87 & 1.04 & 0.84 & 1.00 & $5 / 5$ & 0.87 & 1.04 \\
\hline mpeg2_dec & 2.22 & $5 / 8$ & 2.81 & 1.26 & 2.38 & 1.07 & $5 / 8$ & 4.06 & 1.82 \\
\hline gsm_enc & 2.01 & $7 / 7$ & 4.21 & 2.09 & 3.33 & 1.65 & $2 / 7$ & 4.51 & 2.24 \\
\hline gsm_dec & 1.27 & $2 / 3$ & 6.41 & 5.03 & 2.10 & 1.65 & $1 / 3$ & 6.57 & 5.16 \\
\hline epic_enc & 0.56 & $1 / 2$ & 3.64 & 6.46 & 3.80 & 6.74 & $0 / 2$ & 3.80 & 6.74 \\
\hline epic_dec & 0.81 & $4 / 5$ & 2.97 & 3.67 & 1.83 & 2.26 & $2 / 5$ & 2.97 & 3.67 \\
\hline sum/average & 1.57 & $32 / 40$ & 4.24 & 3.18 & 3.12 & 2.42 & $17 / 40$ & 4.59 & 3.37 \\
\hline
\end{tabular}

${ }^{a} O P C$, operations per cycle; $S P$, speed-up; \#T/L, number of loops with test.

\subsection{Interprocedural Pointer Analysis Evaluation}

5.2.1 Loop-Level Analysis. As complex pointer references is the main issue targeted by DMIT, it is of interest to compare it against advanced interprocedural pointer-analysis techniques. Moreover, as they are not exclusive techniques, we also report the results obtained when using a combination of both. That is, static Pcode-interprocedural analysis is first applied at the front-end. DMIT is then used before the scheduling to disambiguate those loops that have not been previously disambiguated.

Table IV shows the operations per cycle rate and the speed-up achieved at loop level by each compilation model. For the models that include DMIT, we also show the number of loops that require the dynamic test to be disambiguated.

In general, we observe that DMIT achieves better results than IPA (1.31X speed-up over IPA in average), even though it requires lower implementation complexity. After interprocedural pointer analysis, the test block is avoided for 15 loops. However, loop duplication is still performed to 17 of the remaining loops after pointer analysis, which means that dynamic information is still needed to determine the existence or not of the dependence in those loops.

Furthermore, most of the benchmarks exhibit a beneficial effect when both techniques are used together. In mpeg2_dec, for example, the DMIT succeeds in disambiguating five loops (_form_comp. $x$ and _Fast_IDCT.5), which pointer analysis does not, while pointer analysis is able to disambiguate another one (_AddBlock.42). This loop uses a table to perform saturation, and DMIT is unable to deal with this kind of non-strided references.

We have also observed that Pcode IPA lacks array-dependence analysis for reference groups. Six loops achieve significant gains over pointer analysis without doing the test. All these loops are examples of reference groups. For example, in the _ycc_rgb_c.5 (which is the reverse process to the color conversion example in Section 3.4), there are three loads from independent input streams and three stores to consecutive elements of the output stream. IPA succeeds in disambiguating the three loads from the three stores, but it fails to disambiguate the three stores. In this case, our simple static reference group optimization provides an additional speed-up of 1.16X over IPA. 


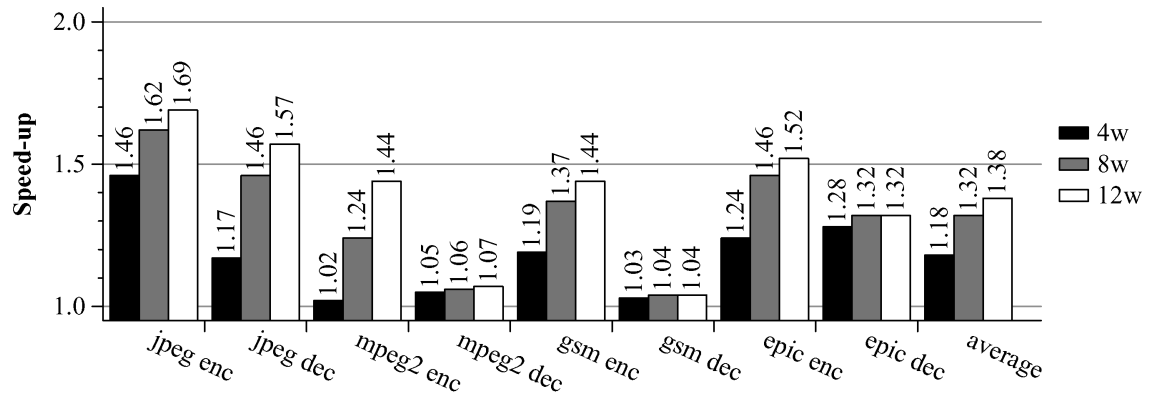

(a) IPA

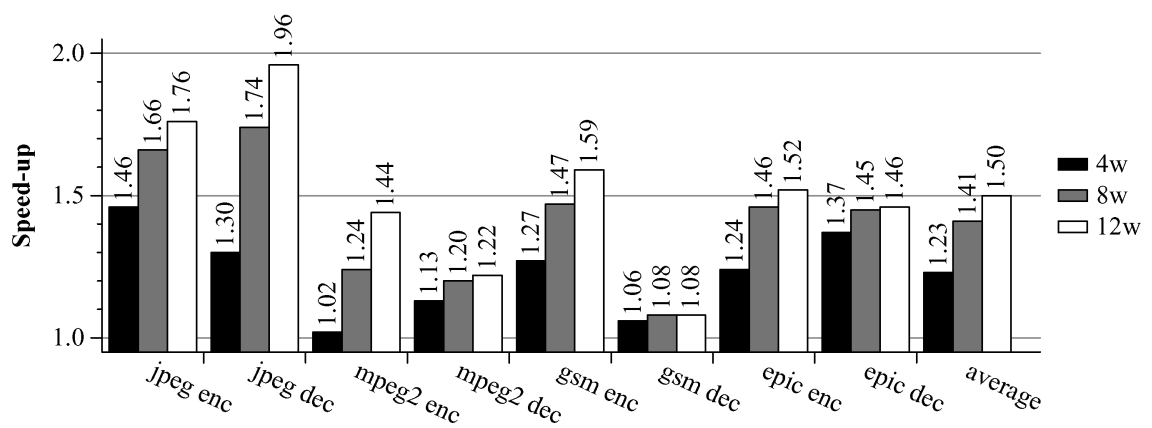

(b) IPA + DMIT

Fig. 9. Performance speed-up of 4-, 8- and 12-issue width architectures with IPA and IPA+ DMIT over the 4 -issue width baseline.

On the other hand, five loops fail the test at runtime with and without pointer analysis. Their dependences were probably proved to be certain at the interprocedural pointer analysis phase, but this information is lost before DMIT, so that it can not differentiate between likely and certain dependences. Maintaining this information would be useful to avoid unnecessary tests.

5.2.2 Complete Applications Speed-up. One advantage of IPA is that it is performed at the beginning of the compilation process, so that it can provide useful information to other phases of code optimization, such as loop invariantcode removal. On the contrary, DMIT is only applied to a fraction of the code and it only aids the scheduling process.

Figure 9(a) shows the speed-up obtained for the 4-, 8-, and 12-issue width architectures over the 4-issue width baseline. Although DMIT outperforms IPA in an average $23.6 \%$ in the targeted loops, these loops are only a $36 \%$ of the overall execution time. IPA improves the remaining $64 \%$ of the application in about $1.4 \%$. As a result, the average gains obtained in complete applications with IPA (1.18X, 1.32X, and 1.38X) are very similar to those obtained with DMIT (1.17X, 1.33X, and 1.41X). The average speed-up increase when both techniques are used together (1.23X, 1.41X, and 1.50X).

To facilitate the comparison, Figure 10 shows the speed-up achieved by the three options over the original compiler for the 8-issue width architecture. The 


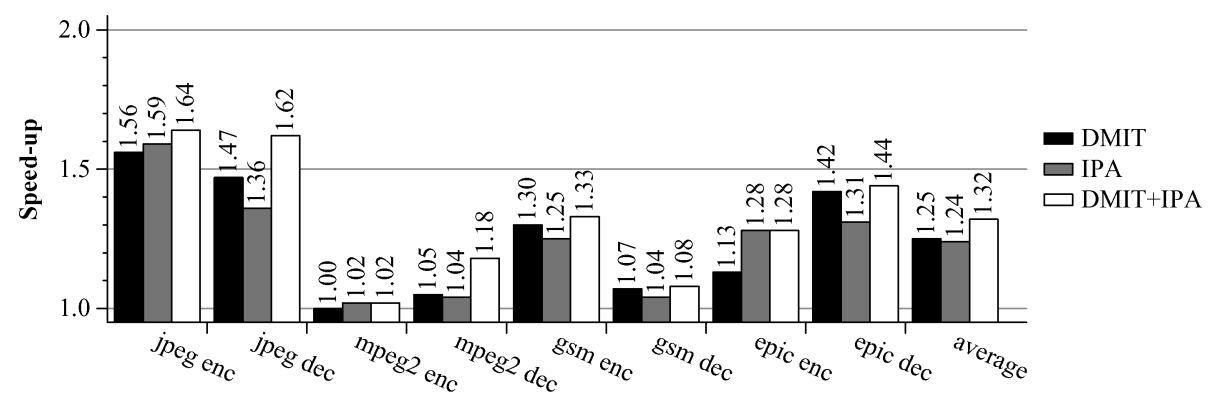

Fig. 10. Performance speed-up of DMIT, IPA, and both over the 8-issue width baseline.

benchmarks do not show a regular behavior. Although, on average, they perform similarly DMIT outperforms interprocedural pointer analysis for five of the eight benchmarks, but it does worse in the remaining three. More interesting are the additional gains obtained with the combination of both, specially for the jpeg_dec and the mpeg2_dec applications.

\section{SUMMARY}

Memory disambiguation of multimedia applications is compromised by the fact that they are often written in languages that support pointer referencing, such as $\mathrm{C}$ or $\mathrm{C}++$. In this paper, we have evaluated a simple, but efficient, memory-disambiguation technique specifically targeted at multimedia loops, or any other kind of applications with similar memory-access patterns.

Taking into account the disjointed behavior of common multimedia memory streams, our algorithm is able to evaluate at runtime whether or not the full loop is disambiguated and execute the corresponding loop version. By calculating the dynamic-memory intervals of every memory reference in a very efficient way at runtime, we avoid having to perform comparisons inside every loop iteration.

In contrast with other dynamic approaches, the DMIT does not require any additional hardware or instructions. It has negligible effects over compilation time and code size, and near-zero cost for all those loops without potential for disambiguation. However, one current limitation of this analysis is the inability to deal with nonstreaming data structures.

Experimental results also confirm that memory disambiguation is a key technique for exploiting the inherent parallelism of multimedia applications. The DMIT provides significant performance gains for a 4-issue width VLIW configuration for most of our benchmarks. Moreover, it allows performance scalability of wider-issue machines in sharp contrast with our baseline.

Although the DMIT outperforms Pcode-interprocedural analysis at the loop level, they perform similarly when we consider full applications. This can be explained by the fact that pointer analysis has the advantage of being applied to the full program code (not only to modulo scheduling loops) and at the beginning of the compilation, so that the alias analysis information can be used by further stages of the process. On the other hand, Pcode pointer analysis lacks arraydependence analysis, which could be overcome with simple static optimizations (such as the detection of reference groups). 
Furthermore, we have shown that a combination of both techniques provides improved results. While there is a significant number of loops for which some information is missing at compile-time, they still benefit from DMIT after interprocedural pointer analysis. On the other hand, the test overhead is avoided for those loops that can be statistically disambiguated.

\section{ACKNOWLEDGMENTS}

We thank Jesús Corbal for his collaboration and Daniel A. Jiménez for his assistance in the preparation of the final version of this paper. This work has been supported by the Ministry of Science and Technology of Spain and the European Union (FEDER funds) under contract TIN2004-07739-C02-01 and by the European HiPEAC network of Excellence. We also wish to thank the Supercomputing Center of Catalonia (CESCA) for supplying the computing resources for our research.

\section{REFERENCES}

Aditya, S., Kathail, V., AND RaU, B. R. 1998. Elcor's machine description system: Version 3.0. Technical Report HPL-98-128, Information Technology Center.

Andersen, L. O. 1994. Program Analysis and Specialization for the C Programming Language. $\mathrm{PhD}$ thesis, DIKU, University of Copenhagen.

Bernstein, D., Cohen, D., And Maydan, D. E. 1994. Dynamic memory disambiguation for array references. In Proceedings of the 27th Annual International Symposium on Microarchitecture. 105-111.

Blume, W. and Eigenmann, R. 1994. The range test: a dependence test for symbolic, non-linear expressions. In Proceedings of the 1994 Conference on Supercomputing. 528-537.

CarR, S., McKinley, K. S., ANd Tseng, C.-W. 1994. Compiler optimizations for improving data locality. In Proceedings of the 6th International Conference on Architectural Support for Programming Languages and Operating Systems. 252-262.

CHOI, J.-D., BuRKe, M., AND CARINI, P. 1993. Efficient flow-sensitive interprocedural computation of pointer-induced aliases and side effects. In Proceedings of the 20th ACM SIGPLAN-SIGACT Symposium on Principles of Programming Languages (Charleston, SC). 232-245.

Emami, M., GhiYa, R., ANd Hendren, L. J. 1994. Context-sensitive interprocedural points-to analysis in the presence of function pointers. In Proceedings of the ACM SIGPLAN'94 Conference on Programming Language Design and Implementation. 242-256.

FEaUtrier, P. 1991. Dataflow analysis of array and scalar references. International Journal of Parallel Programming 20, 1, 23-53.

Gallagher, D. M. 1995. Memory Disambiguation to Facilitate Instruction-Level Parallelism Compilation. PhD thesis, Dept. of Electrical and Computer Engineering, University of Illinois.

Gallagher, D. M., Chen, W. Y., Mahlke, S. A., Grllenhaal, J. C., and Hwu, W. W. 1994. Dynamic memory disambiguation using the memory conflict buffer. ACM SIGPLAN Notices 29, 11, 183193.

Goff, G., Kennedy, K., And Tseng, C. 1991. Practical dependence testing. In Proceedings of the ACM SIGPLAN`91 Conference on Programming Language Design and Implementation. 15-29.

Hind, M. ANd Pioli, A. 2000. Which pointer analysis should I use? In Proceedings of the 2000 ACM SIGSOFT International Symposium on Software Testing and Analysis. 113-123.

Huang, A., Slavenburg, G., AND Shen, J. 1994. Speculative disambiguation: A compilation technique for dynamic memory disambiguation. In Proceedings of the 21st International Symposium on Computer Architecture. 200-210.

Hwu, W. W., Mahlke, S. A., Chen, W. Y., Chang, P. P., Warter, N. J., Bringmann, R. A., Ouellette, R. G., Hank, R. E., Kiyohara, T., HaAb, G. E., Holm, J. G., and Lavery, D. M. 1993. The superblock: An effective technique for vliw and superscalar compilation. The J. Supercomputing 7 , $229-248$.

ACM Transactions on Architecture and Code Optimization, Vol. 2, No. 2, June 2005. 
Hwu, W. W., Hank, R., Gallagher, D., Mahlke, S., Lavery, D., Haab, G., Gyllenhaal, J., and August, D. 1995. Compiler technology for future microprocessors. Proceedings of the IEEE 83, 625-1640.

Kathail, V., Schlansker, M., and RaU, B. R. 2000. Hpl-pd architecture specification: Version 1.1. Technical Report HPL-93-80(R.1), Hewlett-Packard Lab.

LaB., H. P., Group, R.-I., AND Group, I. 1998. Trimaran user manual. http://www.trimaran.org/docs.html.

LANDI, W. 1992. Undecidability of static analysis. ACM Lett. Programming Languages Systems 1, 4 (Dec.), 323-337.

LANDI, W. AND RYDER, B. G. 1992. A safe approximate algorithm for interprocedural pointer aliasing. SIGPLAN Notices 27, 7 (June), 235-248.

Lee, C., Potkonjak, M., and Mangione-Smith, W. H. 1997. Mediabench: A tool for evaluating and synthesizing multimedia and communicatons systems. In Proceedings of the 30th International Symposium on Microarchitecture. 330-335.

Mahlke, S. A., Lin, D. C., Chen, W. Y., Hank, R. E., And Bringmann, R. A. 1992. Effective compiler support for predicated execution using the hyperblock. In Proceedings of the 25th International Symposium on Microarchitecture. 45-54.

Maydan, D., Hennessy, J., AND LAM, M. 1991. Efficient and exact data dependence analysis. In Proceedings of the ACM SIGPLAN'91 Conference on Programming Language Design and Implementation. 1-14.

Moon, S. AND Hall, M. W. 1999. Evaluation of predicated array data-flow analysis for automatic parallelization. In Proceedings of the ACM Symposium on Principles Practice of Parallel Programming. 84-95.

NicolaU, A. 1989. Run-time disambiguation: Coping with statically unpredictable dependencies. IEEE Trans. Computers 38, 5 (May), 663-678.

Paek, Y., Hoeflinger, J., AND PAdUA, D. 1998. Simplification of array access patterns for compiler optimizations. In Proceedings of the ACM SIGPLAN'98 Conference on Programming Language Design and Implementation. 60-71.

Pugh, W. And Wonnacott, D. 1998. Constraint-based array dependence analysis. ACM Transactions on Programming Languages and Systems 20, 3 (May), 635-678.

Ramalingam, G. 1994. The undecidability of aliasing. ACM Trans. Programming Languages and Systems 16, 5 (Sept.), 1467-1471.

RAU, B. R. 1995. Iterative modulo scheduling. Technical Report HPL-94-115, Hewlett-Packard Lab.

Rauchwerger, L. and Padua, D. 1994. The PRIVATIZING DOALL test: A run-time technique for DOALL loop identification and array privatization. In Proceedings of the ACM International Conference on Supercomputing (Manchester, England).

Salamí, E., Corbal, J., Alvarez, C., and Valero, M. 2002. Cost effective memory disambiguation for multimedia codes. In Proceedings of the International Conference on Compilers, Architecture, and Synthesis for Embedded Systems. 117-126.

Salamí, E., Corbal, J., Espasa, R., And Valero, M. 1999. An evaluation of different dlp alternatives for the embedded media domain. In Proceedings of the 1st Workshop on Media Processors and DSPs. 100-109.

Shapiro, M. AND HoRwitz, S. 1997. Fast and accurate flow-insensitive points-to analysis. In Proceedings of the ACM Symposium on Principles of Programming Languages. 1-14.

SteensgaARD, B. 1996. Points-to analysis in almost linear time. In Proceedings of the 23rd ACM SIGPLAN-SIGACT Symposium on Principles of Programming Languages. 32-41.

WiLson, R. P. AND LAM, M. S. 1995. Efficient context-sensitive pointer analysis for c programs. In Proceedings of the ACM SIGPLAN'95 Conference on Programming Language Design and Implementation. 1-12.

Wolf, M. E. AND LAM, M. S. 1991. A data locality optimizing algorithm. In Proceedings of the ACM SIGPLAN'91 Conference on Programming Language Design and Implementation. 30-44.

Yong, S. H., Horwitz, S., AND RePs, T. W. 1999. Pointer analysis for programs with structures and casting. In SIGPLAN Conference on Programming Language Design and Implementation. 91-103.

Received November 2004; revised April 2005; accepted May 2005 